\title{
Recurrent Dedifferentiated Liposarcoma
}

National Cancer Institute

\section{Source}

National Cancer Institute. Recurrent Dedifferentiated Liposarcoma. NCI Thesaurus. Code C150592.

The reemergence of dedifferentiated liposarcoma after a period of remission. 